\title{
Enigma of apnea test for brain death on ECMO-an ongoing discussion-case study and review of literature
}

\author{
Manoj Kumar Sahu ${ }^{1} \cdot$ Prateek Vaswani $^{1} \cdot$ Chalattil Bipin $^{1} \cdot$ Sarvesh Pal Singh ${ }^{1} \cdot$ Vijay Hadda $^{2}$
}

Received: 2 June 2020 / Revised: 26 June 2020 / Accepted: 2 July 2020 / Published online: 1 September 2020

(C) Indian Association of Cardiovascular-Thoracic Surgeons 2020

\begin{abstract}
Coma, absent brain stem reflexes, and apnea are considered the neurological criteria of brain death. As membrane oxygenator does almost entire gas exchange in patients with severely diseased lungs who are on extracorporeal membrane oxygenation (ECMO), it is not possible to carry out apnea test in routine manner. We described the difficulties we faced conducting the apnea test and the other ancillary tests in our patient on veno-venous ECMO who suffered a major cerebral insult and reviewed the literature of brain death testing in patients on ECMO.
\end{abstract}

Keywords Extracorporeal membrane oxygenation $(E C M O) \cdot$ Brain death $\cdot$ Apnea test $\cdot$ Ancillary tests

\section{Introduction}

Brain death (BD) as described by the American Academy of Neurology is the irreversible cessation of all functions of the entire brain including brainstem which requires proof of $a b-$ sence of brainstem reflexes, cessation of spontaneous breathing (apnea), and irreversible coma of a known cause [1]. Extracorporeal membrane oxygenation (ECMO) use has risen in the past decade owing to wide range of applicability in acute, severe, and reversible cardiac, respiratory, or cardiorespiratory failure [2]. However, the issues in legal confirmation of brain death on ECMO patient prevail $[3,4]$. We describe a case in which ECMO was instituted and difficulties for determining BD are highlighted and further description of the literature is discussed.

Manoj Kumar Sahu

drmanojsahu@gmail.com

1 Intensive Care for CTVS, Department of CTVS, Cardiothoracic and Neurosciences Centre, All India Institute of Medical Sciences, Room No. 5, 7th Floor, Ansari Nagar, New Delhi 110029, India

2 Department of Pulmonary Medicine, All India Institute of Medical Sciences, Ansari Nagar, New Delhi 110029, India

\section{Case report}

A 40-year-old female weighing $120 \mathrm{~kg}$ with pneumonia following H1N1 influenza was transferred to our hospital on mechanical ventilation for worsening respiratory distress. Chest X-ray revealed bilateral diffuse infiltrates. The other parameters confirmed severe respiratory failure. The clinical condition worsened on maximal ventilator support and was complicated by superadded infections when respiratory secretions showed growth of Acinetobacter baumanni. The antibiotics were optimized; however, the declining status demanded institution of veno-venous (VV) ECMO on the 4th day of ICU (intensive care unit) admission in our hospital. The drainage cannula was done in the right femoral vein and return cannula was inserted in the right internal jugular vein. Percutaneous insertion under ultrasound guidance applying Seldinger technique was done.

Her course on ECMO was complicated by multiple episodes of sepsis for which management was instituted as per protocol. She showed promising respiratory improvement very late, only by the 86th day of ECMO run. Over this prolonged ECMO run of 3 months, we had to change the oxygenator twice. The first oxygenator surprisingly lasted for 60 days with adequate oxygenation and was changed when post membrane arterial oxygen concentration started declining. The second oxygenator was changed after the 10th day due to thrombus formation. The change of oxygenator was tolerated well on both the occasions. 
Our patient was fully awake and responsive with spontaneous ventilation on ECMO weaning when an episode of generalized tonic clonic seizure (GTCS) occurred on the 88th day of ECMO support. This GTCS episode was managed by single bolus dose of intravenous midazolam $5 \mathrm{mg}$ and levetiracetam $1000 \mathrm{mg}$ and re-establishment of full ECMO support and mechanical ventilation. There was no repeat episode following this. The patient was not arousable with fixed pupils without any observed motor activity. We did not administer any further sedatives, anti-convulsants, muscle relaxants, or narcotics. The liver and renal functions were in the optimal range. Adequate time of 4-5 half-lives of these agents (in our case $48 \mathrm{~h}$ ) had passed before brain death declaration. We considered waiting additional $48 \mathrm{~h}$ owing to the long ECMO run before it was discontinued on the 92nd day.

Just before the GTCS, her VV ECMO settings (on weaning process) were blood flow rate $2.5 \mathrm{~L} / \mathrm{min}$, fraction of inspired oxygen concentration $\left(\mathrm{FiO}_{2}\right)$ 0.7, and sweep gas flow rate $3.0 \mathrm{~L} / \mathrm{min}$. Her blood pressure (BP) was $114 / 84 \mathrm{mmHg}$ and she was on pressure support $\left(12 \mathrm{~cm} \mathrm{H}_{2} \mathrm{O}\right)$ ventilation with positive end expiratory pressure (PEEP) of $6 \mathrm{~cm} \mathrm{H}_{2} \mathrm{O}$ with spontaneous breathing. Following the GTCS, she became unconscious. ECMO flows were increased to $5.5 \mathrm{~L} / \mathrm{min}$ with sweep gas flow of $6 \mathrm{~L} / \mathrm{min}$ at $\mathrm{FiO}_{2} 1.0$ and changed her ventilation to pressure control mode. As coma persisted even after $24 \mathrm{~h}$ of resuscitation, neurological examination was repeated which confirmed complete unresponsiveness with absent cranial nerve and brainstem reflexes with dilated and non-reactive pupils. Apnea test (AT) was performed for declaration of BD in the following manner - core temperature was maintained at $36{ }^{\circ} \mathrm{C}$, systolic BP was maintained $>110 \mathrm{mmHg}$ with minimal ionotropic support, pre-oxygenation was done for $10 \mathrm{~min}$ by increasing the $\mathrm{FiO}_{2}$ on the membrane oxygenator as well as ventilator to $100 \%$. A baseline arterial blood gas (ABG) showed $\mathrm{pH} 7.33$, arterial partial pressure of carbon dioxide $\left(\mathrm{PaCO}_{2}\right) 38.5 \mathrm{mmHg}$, and arterial partial pressure of oxygen $\left(\mathrm{PaO}_{2}\right) 115 \mathrm{mmHg}$, and oxygen saturation 98\%. The sweep gas flow on ECMO was reduced to $0.5 \mathrm{~L} / \mathrm{min}$ and the mechanical ventilator was disconnected. A suction catheter (12 gauge) was introduced through the tracheostomy tube up to the level of carina and $100 \% \mathrm{O}_{2}$ was insufflated at a flow of $8 \mathrm{~L} / \mathrm{min}$. Two minutes through the AT, the patient desaturated to < $85 \%$, BP decreased to $85 / 50 \mathrm{mmHg}$ and no spontaneous breathing was observed. Hence, AT was aborted and she was connected back on ventilator and sweep gas flow on ECMO was increased to $5 \mathrm{~L} / \mathrm{min}$. A quick repeat $\mathrm{ABG}$ showed $\mathrm{PaCO}_{2}$ $43 \mathrm{mmHg}$ (total increment of $4.5 \mathrm{mmHg}$ from baseline) and $\mathrm{PaO}_{2}$ fell to $54 \mathrm{mmHg}$. There was no observed spontaneous respiratory drive during the period of AT. The test was considered inconclusive. We repeated the AT after $1 \mathrm{~h}$ of oxygenation in similar manner but once again we failed to complete the test due to hemodynamic instability and development of hypoxia. Hence, we decided to apply ancillary tests to document BD.
Computerized tomography (CT) angiography or magnetic resonance imaging (MRI) could not be performed because of inability to move the critically ill patient to radiology suite and also logistic issue of ECMO incompatibility with MRI. Transcranial Doppler (TCD) did not show a good window due to edematous scalp tissue. Electroencephalography (EEG) test was inconclusive due to hostile intensive care unit environment with multiple electrical interferences. Finally based on clinical neurological examination done twice at 24-h intervals, $\mathrm{BD}$ was determined.

The third oxygenator was working fine with adequate oxygenation until we called off the support. The patient's family did not give consent for organ transplantation; hence, it was decided not to consider the patient for organ harvesting. Therefore, the ECMO support was discontinued as a part of DNR (do not resuscitate).

\section{Discussion}

ECMO is a form of extracorporeal life support which acts as a rescue in patients with refractory cardiac and respiratory failure. Expanding indications have provided for its increasing use; however, there has been a noted rise in neurological injury in ECMO patients with an incidence of $7.1 \%$ of which $23.5 \%$ manifest as $\mathrm{BD}[2,5,6]$. These sequelae may result from pre-existing neurological dysfunction, hypoperfusion due to dysfunctional cerebral autoregulation, reperfusion injury post resuscitation, rapid blood gas alteration, micro-emboli, thrombosis, and intracranial hemorrhage due to development of coagulopathy or anticoagulant use and differential hypoxiainduced cerebral dysfunction (Harlequin syndrome) in the case of peripheral venoarterial (VA) ECMO $[2,4]$.

$\mathrm{BD}$ declaration would require demonstration of irreversible unconsciousness, apnea, absent cranial nerve, and brainstem reflexes. To ensure the conduct of AT appropriately, normothermia (core temperature $\geq 36{ }^{\circ} \mathrm{C}$ ) and systolic $\mathrm{BP}>$ $100 \mathrm{mmHg}$ are essential [1]. The other prerequisites are the absence of central nervous system-depressant drugs or neuromuscular blocking agents, severe electrolyte, acid-base, or endocrine disturbances. The test is considered positive if there is rise in $\mathrm{PaCO}_{2} \geq 60 \mathrm{mmHg}$ or at least $20 \mathrm{mmHg}$ rise above baseline. AT is considered negative if there is an observed respiratory movement either visualized during the test, detected by ventilator, or by evidence of thoraco-abdominal de-synchrony in consolidated lungs with poor thoracic compliance. It is abandoned in cases of development of hypoxia or deranged hemodynamics $[3,7]$.

Unlike patients on mechanical ventilation alone, gas exchange for patients supported on ECMO occurs mainly through the membrane oxygenator. Carbon dioxide elimination is dependent on the sweep gas flow rate in the circuit. ECMO alters drug pharmacodynamics due to membrane 
sequestration and interference with decarboxylation, due to which lipophilic drugs may persist for prolonged periods interfering with $\mathrm{BD}$ assessment $[6,7]$. Hepatic and renal dysfunction may further delay the drug clearance. Gaseous exchange across the membrane depends on sweep gas flow, the differential movement of carbon dioxide and oxygen, and differential solubility, and hence, the adequate increase in $\mathrm{CO}_{2}$ either does not occur or takes prolonged period which are complicated by hypoxia or hemodynamic alterations $[3,7$, 8]. Hence, on ECMO, the recommended value of $\mathrm{PaCO}_{2}$ rise for a confirmatory AT may not occur even after the stipulated time of AT of 8 to $10 \mathrm{~min}$. The reduction of sweep gas flows to minimum to reduce carbon dioxide elimination also is futile. The respiratory acidosis in addition may worsen the cardiac output in VA ECMO leading to hypotension. The compromised lung in VV ECMO develops hypoxia before an adequate rise in carbon dioxide is observed.

The success of AT in determining BD on ECMO would depend on:

- Maintenance of stable hemodynamics

- Adequate pre-oxygenation

- Prevention of hypoxia in different ways like tracheal insufflation of oxygen through a catheter, T-piece use, adjusting ventilator settings to $100 \%$ oxygen on pressure assist mode, or use of continuous positive airway pressure (CPAP) support for alveolar recruitment in atelectasis

- Reducing $\mathrm{CO}_{2}$ elimination (to ensure its rise) by decreasing the sweep gas flow on ECMO to a minimum of 0.5$1 \mathrm{~L} / \mathrm{min}$ (essential for avoiding hypoxia) or by providing a gas mixture known as carbogen (mixture of $\mathrm{CO}_{2}$ and $\mathrm{O}_{2}$ ) through the ventilator or addition of $\mathrm{CO}_{2}$ in ECMO circuit $[2,5,6,9]$.

In a large meta-analysis AT could confirm BD in $77 \%$ patients on ECMO [6]. In the rest it failed due to absence of appropriate rise in $\mathrm{CO}_{2}$; development of hypoxic, hemodynamic alterations; and neurologist opinion of questionable reliability of AT on ECMO $[4,10]$.

The presence of these lacunae with AT has focused our attention to ancillary tests. These tests are of two types, first are the ones which measure cerebral blood flow and second are those which test the bio-electric activity [3]. The former includes angiograms either conventional or by $\mathrm{CT}$ scan and MRI, TCD, and nuclear studies. The latter include EEG and evoked potentials. The meta-analysis revealed the EEG use in $62 \%$ cases followed by $\mathrm{CT}$ angiogram in $22 \%$ cases and TCD in $6 \%$ cases [6].

EEG demonstration of electro-cerebral inactivity defined as absence of non-artefactual electrical activity of at least $2 \mu \mathrm{V}$ from baseline over half an hour period is supportive of brain death. The CT angiogram should demonstrate absence of blood flow in internal carotid and vertebral arteries to signify BD. In addition, CT scan may also demonstrate other neurological causes of declining Glasgow coma scale (GCS) such as massive intracranial bleed with raised intracranial pressure and midline shift amenable to urgent neurosurgical intervention. This if left unattended may lead to catastrophic sequelae of herniation culminating in $\mathrm{BD}$.

EEG and evoked potentials are affected by potential confounders such as sedative drug use, hypo- or hyperthermia, metabolic abnormalities, and other local electrical interferences. Bispectral index is used in conjunction, with variable success [10]. The tests of blood flow detection, although, unaffected by these confounders may provide false positive results in significant hypotension states or in cases of occluded proximal vasculature and they show false negative with preserved blood flow in BD patients with craniotomy or ventricular drain in situ. Also, these tests are difficult to perform due to concerns with patient shifting to CT or MRI areas and MRI incompatible ECMO circuits. TCD fails to visualize evidence of cerebral circulatory arrest in majority of adult patients,which however can be used to demonstrate presence of oscillatory flow with systolic spikes in pediatric subset $[3,5,10]$. Ancillary tests on their own cannot be used for conclusive BD declaration, so AT is mandatory $[2,3,6]$. In our patient, AT was tried on 2 occasions but could not be completed, so proved inconclusive.

The facility for serum levels is not available at our center; however, serum levels of sedative agents should be incorporated in the institutional protocol. The elimination half-lives of midazolam being $1.5-2.5 \mathrm{~h}$ and levetiracetam being $6-8 \mathrm{~h}$ were taken into consideration before a formal neurological examination. The other commonly used agents in the ICU with their elimination half-lives are mentioned in Table 1 [11].

Ancillary tests such as EEG were tried but lot of interference pattern was seen; TCD could not demonstrate the flow due to local tissue edema owing to long ECMO period. Borderline hemodynamics with inotropic support and risk of decannulation/ECMO circuit disconnections precluded patient transfer to CT facility.

Hence, the declaration of BD was done in accordance with the neurologist after $48 \mathrm{~h}$ had elapsed to account for delayed drug clearance.
Table 1 Elimination half-lives of commonly used sedative and narcotic agents in the intensive care unit 
In spite of all the shortcomings described in the case about conduction of clinical test including the AT and the ancillary tests (EEG and CT/MRI) for evaluating BD, in the present era, these tests are essential to conclude BD and should be a part of the institutional protocol; however, the logistic issues at our center did not permit us to do so. The subjective nature of opinion by neurologist should also be kept in mind highlighting the need for the ancillary test in cases with failed AT for BD determination. Serum levels of the narcotic agents should be estimated in such cases whenever feasible.

This case and review focuses the attention on the dire need to modify AT for aiding the conclusive diagnosis of $\mathrm{BD}$ in these patients.

\section{Conclusion}

The widened implications with surge in use of ECMO have necessitated the need for establishment of guidelines for BD declaration on ECMO. AT is inconclusive in patients on ECMO owing to the altered physiology and underlying pulmonary conditions for which ECMO was instituted. Ancillary tests may play a complimentary role, which however would still require AT to confirm BD. Implementation of a standardized approach for AT in this subset would aid in establishing a diagnosis of $\mathrm{BD}$ in timely manner so that organ donation could be facilitated appropriately.

\section{Funding None.}

\section{Compliance with ethical standards}

Conflict of interest The author(s) declare that there are no potential conflicts of interests that are directly or indirectly related to this publication, financial or otherwise.

Ethics approval Not applicable
Informed consent Informed consent has been taken from the next of kin to publish this case report.

\section{References}

1. Wijdicks EF. Determining brain death in adults. Neurology. 1995;45:1003-11.

2. Lie SA, Hwang NC. Challenges of brain death and apnea testing in adult patients on extracorporeal membrane oxygenation-a review. $J$ Cardiothorac Vasc Anesth. 2019;33:2266-72.

3. Kreitler KJ, Cavarocchi NC, Hirose H, et al. Declaring Brain Death on ECMO. JHN J. 2017;12:30-4.

4. Muralidharan R, Mateen FJ, Shinohara RT, Schears GJ, Wijdicks EFM. The challenges with brain death determination in adult patients on extracorporeal membrane oxygenation. Neurocrit Care. 2011;14:423-6.

5. Meadows C, Toolan M, Slack A, et al. Diagnosis of death using neurological criteria in adult patients on extracorporeal membrane oxygenation: Development of UK guidance. J Intensive Care Soc. 2020;21:28-32.

6. Migdady I, Stephens RS, Price C, Geocadin RG, Whitman G, Cho SM. The use of apnea test and brain death determination in patients on extracorporeal membrane oxygenation: A systematic review. $J$ Thorac Cardiovasc Surg. 2020; in press.

7. Beam WB, Scott PD, Wijdicks EFM. The Physiology of the apnea test for brain death determination in ECMO: arguments for blending carbon dioxide. Neurocrit Care. 2019;31:567-72.

8. Madden M, Andrews P, Rector R, Menaker J, Habashi N. Carbogen for apnea testing during the brain death declaration process in subjects on extracorporeal membrane oxygenation. Respir Care. 2020;65:75-81.

9. Smilevitch P, Lonjaret L, Fourcade O, Geeraerts T. Apnea test for brain death determination in a patient on extracorporeal membrane oxygenation. Neurocrit Care. 2013;19: 215-7.

10. Jarrah RJ, Ajizian SJ, Agarwal S, Copus SC, Nakagawa TA. Developing a standard method for apnea testing in the determination of brain death for patients on venoarterial extracorporeal membrane oxygenation: a pediatric case series. Pediatr Crit Care Med. 2014;15:e38-43.

11. Peck TE, Hill SA, Williams M. Pharmacology for anaesthesia and intensive care. $3^{\text {rd }}$ ed. Cambridge, UK: Cambridge University Press; 2008.

Publisher's note Springer Nature remains neutral with regard to jurisdictional claims in published maps and institutional affiliations. 